\title{
Interferon- $\boldsymbol{\gamma}$ induced nitric oxide mediates in vitro neuronal damage by Trypanosoma cruzi-infected macrophages
}

\author{
Camila Megale de Almeida-Leite, ${ }^{\mathrm{a}}$ Lúcia Maria da Cunha Galvão, ${ }^{\mathrm{b}}$ Luis C.C. Afonso, \\ Fernando de Queiróz Cunha, ${ }^{\mathrm{d}}$ and Rosa Maria Esteves Arantes ${ }^{\mathrm{a}, *}$ \\ ${ }^{a}$ Department of Pathology, Institute of Biological Sciences, Federal University of Minas Gerais, Belo Horizonte, Minas Gerais, Brazil \\ ${ }^{\mathrm{b}}$ Department of Parasitology, Institute of Biological Sciences, Federal University of Minas Gerais, Belo Horizonte, Minas Gerais, Brazil \\ ${ }^{\mathrm{c}}$ Department of Biological Sciences, Federal University of Ouro Preto, Ouro Preto, Minas Gerais, Brazil \\ ${ }^{\mathrm{d}}$ Department of Pharmacology, Faculty of Medicine of Ribeirão Preto, University of São Paulo, Ribeirão Preto, São Paulo, Brazil
}

Received 2 June 2006; revised 1 September 2006; accepted 5 September 2006

Available online 20 October 2006

\begin{abstract}
Neuronal lesions and peripheral denervation in Chagas' disease are related to local inflammation; however, the pathogenic mechanisms of neuronal lesions in the heart and megavisceras are still unclear. We investigated the involvement of nitric oxide (NO) on neuronal lesion in co-cultures of neurons and macrophages. Trypanosoma cruzi-infected and interferon- $\boldsymbol{\gamma}(\mathrm{IFN}-\boldsymbol{\gamma})$-activated co-cultures of neurons and wildtype (WT) macrophages showed significant reduction of both neuronal survival and neurite density. These findings correlated with the levels of NO and the expression of inducible nitric oxide synthase (iNOS). Accordingly, neuronal survival rate in the co-cultures was recovered to control levels by treatment of the cultures with the iNOS inhibitor, aminoguanidine. Moreover, neither neuronal survival nor the neurite density was affected in the co-cultures when the macrophages were harvested from iNOS-deficient mice. These results demonstrate that iNOS-derived $\mathrm{NO}$ is the major molecule involved in neuronal damage mechanism in our in vitro model of Chagas' disease neuropathology. (C) 2006 Elsevier Inc. All rights reserved.
\end{abstract}

Keywords: SCG; Co-cultures; T. cruzi; Nitric oxide; Neuron; IFN- $\gamma$; iNOS; Macrophage; Chagas' disease

\section{Introduction}

Despite recent progress in the control of Chagas' disease, approximately 20 million individuals are still chronically infected with its causative agent, Trypanosoma cruzi. Indeed, Chagas' disease is a significant cause of morbidity and mortality in Latin America (Moncayo, 2003). Infection with $T$. cruzi presents initially

\footnotetext{
* Corresponding author. Departamento de Patologia Geral, Instituto de Ciências Biológicas, Universidade Federal de Minas Gerais, Av. Antônio Carlos, 6627, 31270-901, Belo Horizonte, MG, Brazil. Fax: +5531 3499 2879.

E-mail address: rosa@icb.ufmg.br (R.M.E. Arantes).

Available online on ScienceDirect (www.sciencedirect.com).
}

as an acute phase, during which the parasite infects a wide variety of tissues. In most patients, specific host defenses develop and the acute phase subsides without any serious sequelae. The acute phase passes into a chronic phase, in which a few parasites remain in the circulating blood and body tissues, though most patients remain asymptomatic at this stage. However, some individuals develop clinical manifestations 10 to 30 years after the onset of infection (Rossi and Bestetti, 1995). These typically present as cardiac arrhythmias, cardiomyopathy or disturbances in esophageal and colonic motility (Köberle, 1968) involving hypertrophy and dilatation of the organ, i.e., mega esophagus and mega colon. The underlying anatomical abnormality in the organs appears to be a reduction in ganglion cell number (Köberle, 1970). In the past, several hypotheses were investigated in order to explain the mechanism of neuronal damage. Indeed, the existence of a neurotoxic agent liberated by the disintegration of the amastigote forms (Köberle, 1956; Alvarenga, 1960; MacClure and Poche, 1960) and the direct destruction of nerve cells through parasitism (Okumura, 1966; Köberle, 1968) were proposed but never proven. Similarly, myenteric denervation has been attributed to an intense inflammatory reaction around the ruptured pseudocysts (Tafuri, 1970).

Recently, we described the loss of enteric ganglia in T. cruzitriggered tissue inflammation in a mouse model of Chagas' disease. Our in vivo studies of mice lacking the inducible nitric oxide synthase (iNOS) gene indicated that the acute phase of $T$. cruzi infection led to myenteric denervation due to IFN- $\gamma$ elicited nitric oxide (NO) production resulting from iNOS activation in the inflammatory foci along the intestinal wall (Arantes et al., 2004). We interpreted these data as an indirect evidence for the role of iNOS in the mechanism of neuronal damage in this disease. However, interactions between inflammatory cells and nerves are complex and difficult to assess in vivo. In particular, the specific sources of the several potential mediators of neuronal cell damage that are liberated in these sites of inflammation cannot be clearly resolved.

The induction of iNOS by diverse stimuli, such as microbial and/or cytokines, is $\mathrm{Ca}^{2+}$ independent and results in the generation 
of high and enduring NO levels (Nathan and Xie, 1994; Stuehr, 1999) in many cell types including macrophages, muscle cells, hepatocytes, fibroblasts, astrocytes and endothelial cells (reviewed in Nathan and Shiloh, 2000). It has been demonstrated that resistance to $T$. cruzi infection is associated with the capacity of lymphocytes to generate IFN- $\gamma$, which in turn can activate macrophages to produce $\mathrm{NO}$, the main effector molecule that controls intracellular T. cruzi multiplication (Gazzinelli et al., 1992; Vespa et al., 1994). Studies have suggested that NO also plays a role in the tissue damage seen in Chagas' disease (Garcia et al., 1999; Vespa et al., 1994; Ny et al., 1999; Pinto et al., 2002; Chandra et al., 2002), but a direct role of macrophage-derived NO in mediating the neurotoxicity of $T$. cruzi was never definitively demonstrated.

Earlier, we described a co-culture model of sympathetic cervical ganglia (SCG) neurons and macrophages, shown to be a useful system for clarifying the roles of inflammatory cells and their products in neuronal injury (Arantes et al., 2000). Using this co-culture model, we have now investigated the direct effect of NO derived from $T$. cruzi-infected and IFN- $\gamma$-activated macrophages on neuronal structure in vitro. Peritoneal macrophages from either normal mice or iNOS knockout $\left(\mathrm{iNOS}^{-/}\right.$) mice were activated in vitro to precisely evaluate the role of macrophage derived nitric oxide in our model of neuronal injury.

\section{Materials and methods}

\section{Culture of SCG neurons}

Pure primary cultures of SCG neurons were prepared as previously described (Arantes et al., 2000; Blennerhassett and Bienenstock, 1998). Briefly, SCG were removed from 2- to 3day-old C57BL/6 (WT) mice and enzymatically dissociated in $0.25 \%$ trypsin II (Sigma Chemical Company, MO). Isolated sympathetic neurons in a final concentration of $10^{4}$ cells/well were cultured on 96-well plates (Sarstedt) coated with Matrigel (Becton Dickinson, NJ) in $50 \mathrm{ng} / \mathrm{ml} \mathrm{NGF} \mathrm{(NGF,} \mathrm{Alomone,}$ Israel), $10 \%$ fetal bovine serum (FBS)/minimal essential medium (MEM) with $2 \times 10^{-6} \mathrm{M}$ cytosine arabinoside (Ara-C, Sigma Chemical Company, MO) to remove glial cells. The remaining accessory cells represented less than $5 \%$ of cells in the cultures as shown in Figs. $1 \mathrm{~A}$ and $\mathrm{H}$ (low magnification fields). The cultures were maintained at $37^{\circ} \mathrm{C}$ in a humidified $5 \% \mathrm{CO}_{2}$ incubator for $48 \mathrm{~h}$ before interventions were made. The identity of neurons was confirmed using the pan neuronal antibody 9.5 Protein Gene Product (PGP 9.5) as described below.

\section{Neuron and macrophage co-cultures}

Neuronal cultures, obtained as described above, were maintained for $48 \mathrm{~h}$ before macrophages were added in order to establish the co-cultures. Macrophages were harvested from the peritoneal cavity of adult WT and $\mathrm{iNOS}^{-/-}$mice and centrifuged at $400 \times \mathrm{g}$ for $10 \mathrm{~min}$. The pellet was resuspended in medium and a single hypotonic lysis was performed as previously described (du Manoir et al., 2002) to remove erythrocytes. After another centrifugation, the cells were resuspended in complete medium and added to the neuronal cultures in a ratio of 10 macrophages per neuron. After $2 \mathrm{~h}$, the cultures were washed to remove nonadherent cells, leaving a $97 \%$ pure population of adherent macrophages.

\section{Parasites}

The Y strain of T. cruzi was used for all experiments. Trypomastigote forms were cultured and purified from monkey kidney epithelial cell line VERO, as previously described (Braga et al., 1993). Parasites were harvested after 6 days in culture, centrifuged at $150 \times \mathrm{g}$ for $10 \mathrm{~min}$ at room temperature, counted in a Neubauer chamber, centrifuged at $450 \times g$ for $10 \mathrm{~min}$ at $4{ }^{\circ} \mathrm{C}$, suspended in 10\% FBS supplemented 199 medium and used for infection of cultures and co-cultures.

\section{T. cruzi infection}

Y strain $T$. cruzi trypomastigotes were added in a parasite-tomacrophage ratio of 10:1 to neuronal cultures or co-cultures. The cells were incubated at $37^{\circ} \mathrm{C}$ and $5 \% \mathrm{CO}_{2}$ in the presence or absence of $200 \mathrm{UI} / \mathrm{ml}$ of recombinant murine IFN- $\gamma$.

\section{Blockade of iNOS activity}

In order to define the role of NO derived from iNOS in neuronal lesions, we obtained macrophages from iNOS ${ }^{-1}$ mice similarly as described above. In some experiments, the iNOS inhibitor aminoguanidine (30 and $100 \mu \mathrm{M}, \mathrm{RBI}$, Natick, MA) was used at to block iNOS activity of WT macrophages (Misko et al., 1993).

\section{Assessment of neuronal damage}

Cultures were fixed in $10 \%$ neutral-buffered formalin solution after different time intervals post-infection (p.i.) and stained with 10\% Giemsa (Doles Reagents, Brazil). Neuronal death was quantified by counting the total number of surviving neurons in the whole area of each well and expressed as the percentage of surviving neurons in controls. The neuronal damage was assessed using the fluorescent probe Hoechst $33342(0.2 \mu \mathrm{g} / \mathrm{ml}$; Molecular Probes). This fluorochrome binds specifically to DNA and is used to evaluate nuclear appearance, detecting changes such as DNA clumping into apoptotic bodies. In addition, apoptotic nuclei were detected with a terminal deoxynucleotidyl transferase-mediated UTP nick end labeling (TUNEL) detection kit (TdT-FragEL, DNA Fragmentation Detection Kit, QIA33, Oncogene Research Products). Data obtained were representative of 3-4 independent experiments in triplicates.

\section{Immunocytochemistry and immunofluorescence}

Cultured cells were washed in PBS and permeabilized with $0.25 \%$ Triton $\mathrm{X}-100$ for $10 \mathrm{~min}$ at room temperature. For immunocytochemistry, endogenous peroxidase activity was abolished with $3.5 \% \mathrm{H}_{2} \mathrm{O}_{2}$ for $30 \mathrm{~min}$, followed by incubation with 1:40 normal goat serum (NGS) for $30 \mathrm{~min}$ at room temperature. The cells were incubated with the primary rabbit anti-PGP 9.5 (1:1000; Ultraclone, UK) or primary mouse anti-CD68 (1:150, 556059, BD Biosciences, USA) antibodies for $2 \mathrm{~h}$ at room temperature. Secondary biotinylated antibodies were goat anti-rabbit and antimouse antibodies, followed by streptavidin-peroxidase complexes (DAKO-LSABR 2 system; DAKO, Carpinteria, CA, USA). The reaction was visualized by incubating the cultures with 3,3diaminobenzidine tetrahydrochloride (Sigma Chemical Company, MO). For immunofluorescence, cells were incubated with 1:40 NGS and $2 \%$ bovine serum albumin (BSA) for $30 \mathrm{~min}$ each at room 
temperature. Macrophages and iNOS expression were detected with 1:500 dilution of the FITC-conjugated primary rat anti-F4/80 (MCA497F, Serotec, USA) antibody and 1:50 dilution of primary rabbit anti-iNOS (AB1631, Chemicon, EUA) and Alexa 568conjugated secondary antibodies (1:500; Molecular Probes), respectively. Secondary antibodies alone were used as specificity controls and uniformly resulted in very low background levels of reactivity. The cells were examined by microscopy and digital images were acquired for documentation.

Neurite density assessment

As an additional assessment of neuronal damage, the neurite area were measured in control cultures and at $48 \mathrm{~h}$ p.i. following
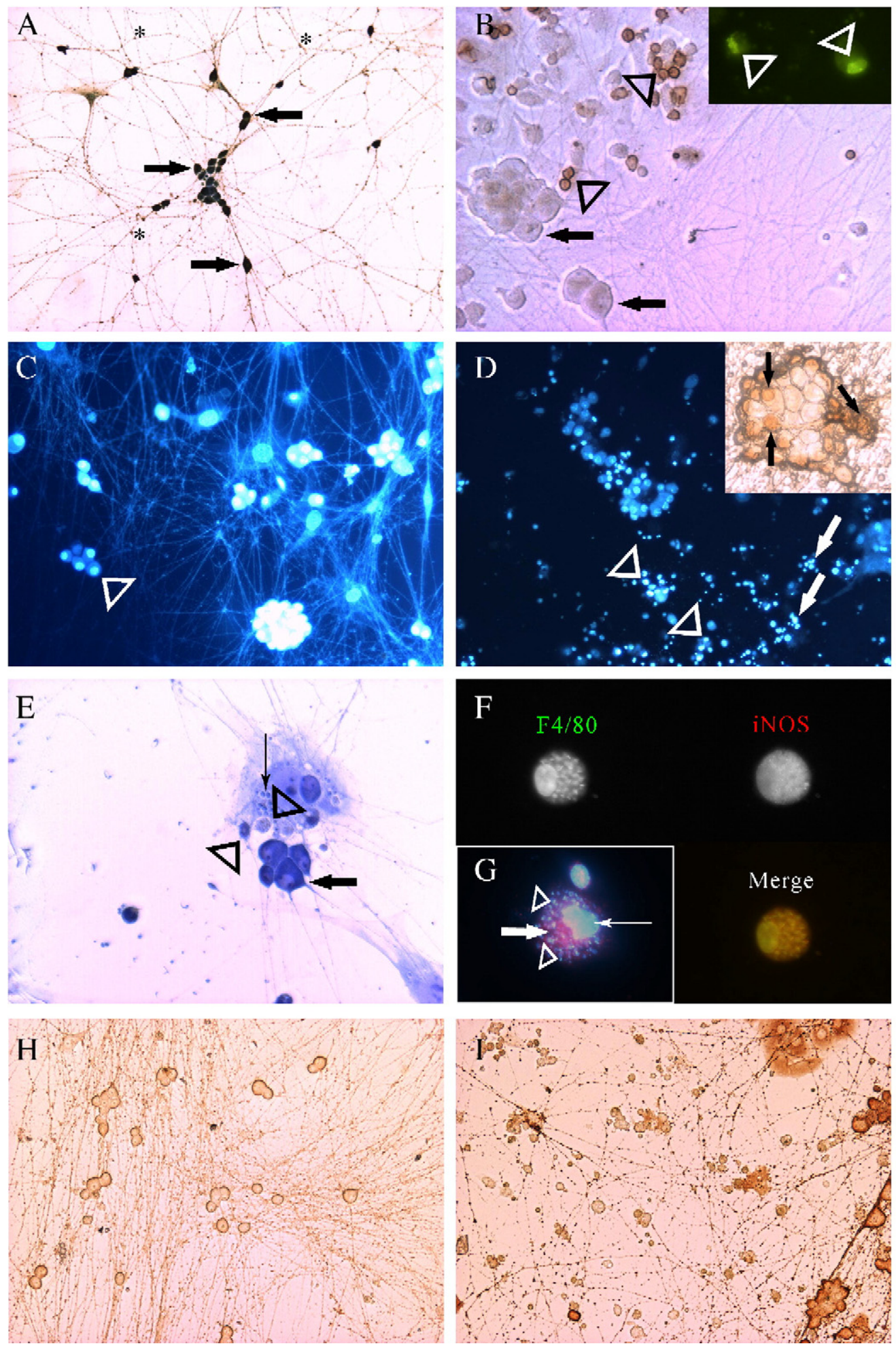
immunocytochemistry for PGP 9.5. Captured video images of culture dishes were measured for the neurite area using the KS300 program (Version 2.0, Zeiss, Germany) and neurite density was expressed as the percentage of neurite area relative to control. Data obtained were representative of 3-4 independent experiments in triplicates.

\section{Evaluation of $\mathrm{NO}^{-2}$ production by macrophages}

Culture supernatants were collected at different times points p.i., stored at $-20^{\circ} \mathrm{C}$ and assayed for the nitrite concentration by mixing $0.1 \mathrm{ml}$ of culture supernatant with $0.1 \mathrm{ml}$ of $(0.1 \%$ naphthylethylene diamine dihydrochloride and $1 \%$ sulfanilamide plus $2.5 \%$ phosphoric acid) as previously described (Green et al., 1981). Absorbance at $540 \mathrm{~nm}$ was read $10 \mathrm{~min}$ later, and $\mathrm{NO}^{-2}$ concentration was determined by reference to a standard curve of 1 to $200 \mu \mathrm{M} \mathrm{NaNO}_{2}$.

\section{Statistical analysis}

The results were expressed as the average and SEM of at least three independent experiments and analyzed for statistical significance with Mann-Whitney $U$ test. Probability values $(P)$ of 0.01 or less were considered significant.

\section{Results}

\section{Aspects of SCG neurons in cultures and co-cultures}

The neurons in vitro conserved their morphological characteristics and displayed extensive neurite outgrowth with visible varicosities at $48 \mathrm{~h}$ post-plating (Fig. 1A), which was evident by the immunoreactivity with the pan-neuronal marker PGP 9.5. Efficient inhibition of glial cell proliferation was maintained by ARA-C.

The addition of pure populations of peritoneal macrophages to neuronal cultures resulted in a homogenous pattern of distribution of CD68 (Fig. 1B) and F4/80 (Fig. 1B, insert) immunopositive macrophages that were dispersed among the neurites.

\section{T. cruzi induced macrophage activation causes neuronal death in co-cultures}

Treatment of neuronal cultures by addition of T. cruzi indicated a lack of direct action of the parasite on neuronal cultures (Fig. 2B). Because no significant neuronal death was observed, we next assessed macrophages for their potential to cause neuronal damage in vitro following infection with T. cruzi and activation with IFN- $\gamma$. This caused progressive and extensive neuronal death, with $73 \%$,
$49 \%$ and $40 \%$ of neuronal survival relative to controls at 24,48 and $72 \mathrm{~h}$ p.i., respectively (Figs. 2A-C). Treatment of co-cultures by addition of $T$. cruzi-infected or IFN- $\gamma$-activated macrophages and treatment of neuronal cultures by IFN- $\gamma$ (Figs. 2A-C) did not significantly affect either neuronal morphology or survival relative to pure neuronal cultures controls at any time points.

In T. cruzi-infected and IFN- $\gamma$-activated co-cultures of neurons and WT macrophages, we observed shrinkage of cellular bodies and irregularities of cell membrane associated with pycnotic nuclei stained by Hoechst 33342 (Fig. 1D) compared to the control aspect showed in Fig. 1C. In addition, apoptotic nuclei were detected by TUNEL labeling (Fig. 1D, insert). In parallel cultures, the identity of each cell was detected as described above (Figs. 1A and B). In these co-cultures, we frequently also identified necrotic and degenerative neuronal changes characterized by cell vacuolization and swollen nuclei, cytoplasmatic dissolution and the presence of cellular debris (Fig. 1E). These very intense alterations led to loss of morphological aspects of the cell types in co-culture (Figs. 1D and E). In contrast, these changes were never seen in pure neuronal cultures or in any of our other co-cultures conditions (Fig. 1C). Close proximity between damaged neurons and parasitized macrophages immunopositive for iNOS was often found (Fig. 1G), suggesting a link between NO generation and subsequent neuronal damage. This physical proximity of neurons and activated macrophages in our model mimics natural proximity of inflammatory cells and tissue damage described in human and experimental Chagas' disease (Tafuri, 1970; da Silveira et al., 2005).

\section{Neurite density}

To evaluate the possibility of a differential effect of macrophage-mediated damage on neuronal cell body vs. neurites under our experimental conditions, neurite area was quantified at $48 \mathrm{~h}$ p.i. (Fig. 3). Neurite density was reduced to $40 \%$ of control neurite area in simultaneously T. cruzi-infected and IFN- $\gamma$-activated co-cultures of neurons and macrophages (Figs. $1 \mathrm{H}$ and I). The direct addition of either T. cruzi or IFN- $\gamma$ to neuronal cultures had no effect on neurite density by $48 \mathrm{~h}$ post-treatment, and again ruled out a direct effect of these agents on neuronal structure (Fig. 3).

\section{Nitric oxide production correlates to neuronal death}

Nitrite levels in culture supernatants were measured by the Griess reaction as an indicator of $\mathrm{NO}$ production in our system. The addition of alternatively $T$. cruzi, IFN- $\gamma$ or peritoneal macrophages to neuronal cultures and treatment of co-cultures by addition of $T$. cruzi-

Fig. 1. (A) Aspects of SCG neurons in cultures stained with the pan-neuronal marker PGP 9.5. Neuronal cell bodies (arrows) and varicosities (*). Original magnification: $\times 35$. (B) Distribution of neurons and WT macrophages in co-cultures. CD68 (open arrowheads)-immunopositive WT peritoneal macrophages in co-culture with non-stained SCG neurons (arrows). Insert: Immunofluorescence of macrophages with F4/80 (green, open arrowheads). Original magnification: $\times 65$. (C and D) Nuclear staining by Hoechst fluorescent probe 33342 (blue) of pure neuronal control cultures (C) and of WT T. cruzi-infected and IFN- $\gamma-$ activated co-cultures (D). Compare condensed and fragmented nuclei, suggesting apoptotic changes in neurons in panel D (open arrow heads) with normal nuclei aspect in control (C, open arrow heads). Notice the presence of cell debris in WT T. cruzi-infected and IFN- $\gamma$-activated co-cultures (D, white arrows). Insert: Apoptotic neuronal death in co-culture demonstrated by TUNEL labeling of some neurons (black arrows) in WT T. cruzi-infected and IFN- $\gamma$-activated cocultures. Original magnifications: $\times 35(\mathrm{C}$ and $\mathrm{D}) ; \times 65(\mathrm{D}$, insert). (E) Some intact (arrow) and intensely degenerated neurons $($ arrowheads) in close proximity to intracellular amastigotes (long arrow) and cell debris. Giemsa staining in WT T. cruzi-infected and IFN- $\gamma$-activated co-cultures. Original magnification: $\times 65$. (F) F4/80 (green) and iNOS (red) immunopositivity in WT T. cruzi-infected and IFN- $\gamma$-activated macrophages were merged in lower panel. Original magnification: $\times 125$. (G) Nuclear staining by Hoechst fluorescent probe 33342 (long arrow, blue) and iNOS immunopositivity (arrow, cytoplasmatic staining in red) of WT $T$. cruzi-infected and IFN- $\gamma$-activated macrophages. Notice H33342 staining of amastigotes DNA inside macrophages (blue dots, open arrowheads). Original magnification: $\times 125$. ( $\mathrm{H}$ and $\mathrm{I})$ Representative aspects of neurites stained with the pan-neuronal marker PGP 9.5 in control cultures, normal density $(\mathrm{H})$ and in WT T. cruzi-infected and IFN- $\gamma$-activated co-cultures, decreased density (I). Original magnification: $\times 35$. 
infected or IFN- $\gamma$-activated macrophages did not cause a significant increase in NO production at 24, 48 and $72 \mathrm{~h}$ post-treatment (Fig. 4). However, the addition of IFN- $\gamma$ to the T. cruzi-infected WT cocultures resulted in significantly higher levels of NO production at

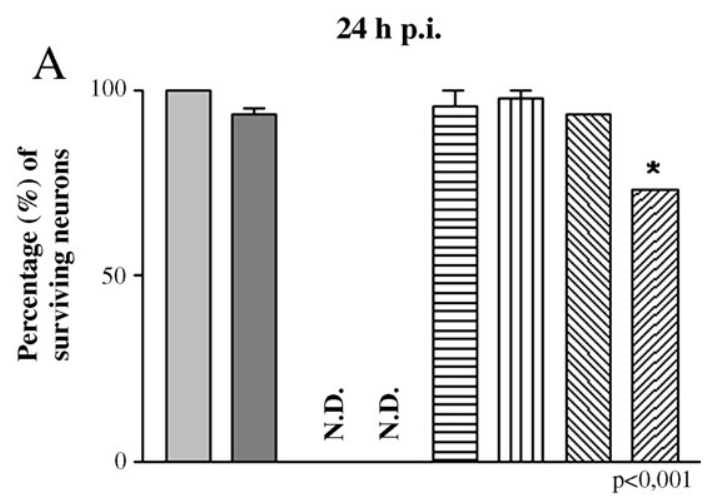

48 h p.i.

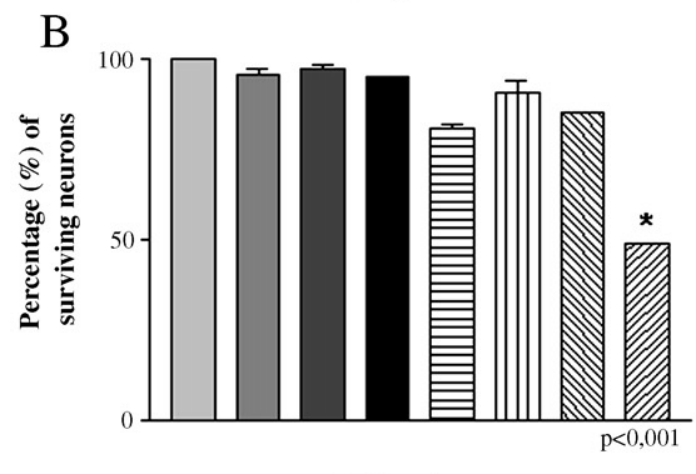

72 h p.i.

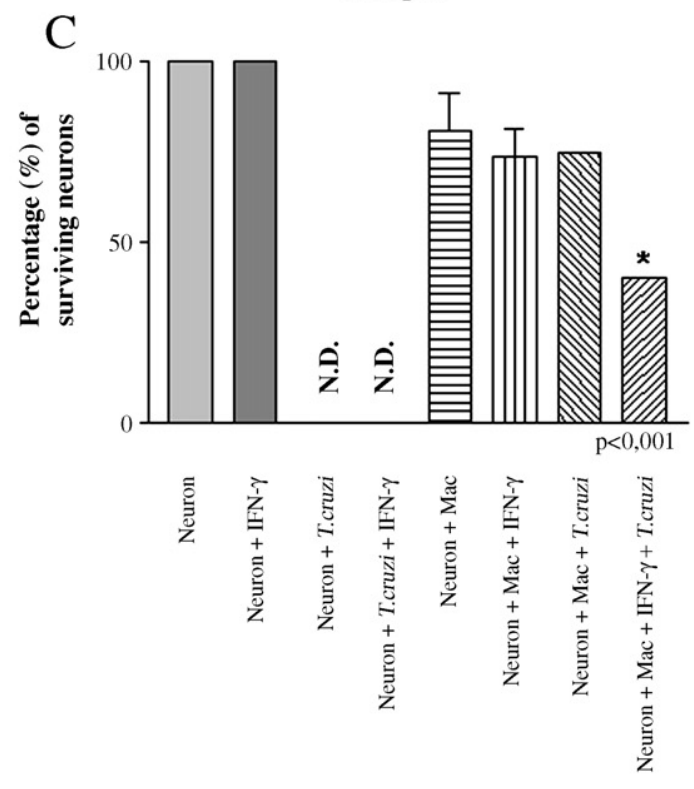

Fig. 2. Decreased neuronal survival in T. cruzi-infected and IFN- $\gamma$-activated co-cultures of neurons and WT macrophages (Neuron $+\mathrm{Mac}+\mathrm{IFN}-\gamma+T$. cruzi) $24 \mathrm{~h}$ (A), $48 \mathrm{~h}$ (B) and $72 \mathrm{~h}$ (C) p.i. We did not detect significant neuronal survival decrease in controls (Neuron, Neuron + IFN- $\gamma$ ) nor experimental (Neuron $+\mathrm{Mac}$, Neuron $+\mathrm{Mac}+\mathrm{IFN}-\gamma, \mathrm{Neuron}+\mathrm{Mac}+T$. cruzi, Neuron $+T$. cruzi, Neuron $+T$. cruzi + IFN- $\gamma$ ) conditions. $N=3$ independent experiments in triplicates per experimental condition, except for Neuron $+T$. cruzi $+\operatorname{IFN}-\gamma(N=3)$. N.D., not done. ${ }^{*} p<0.001$.

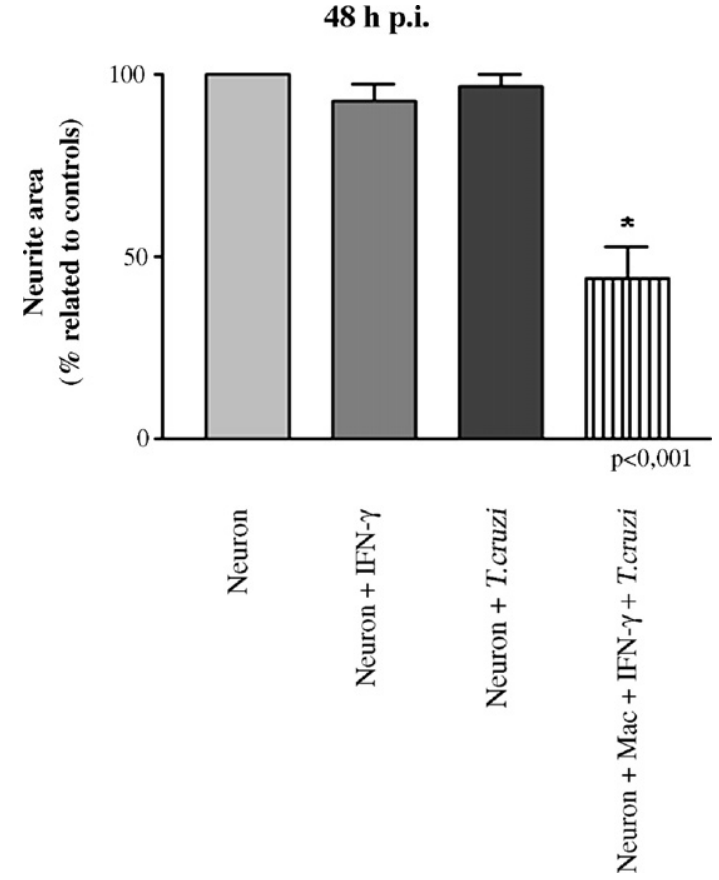

Fig. 3. Decreased neurite density in T. cruzi-infected and IFN- $\gamma$-activated cocultures of neurons and WT macrophages (Neuron $+\mathrm{Mac}+\mathrm{IFN}-\gamma+T$. cruzi) compared to normal density in controls (Neuron, Neuron + IFN- $\gamma$ ) and experimental (Neuron $+T$. cruzi) condition at $48 \mathrm{~h}$ p.i. $N=3$ independent experiments in triplicates per experimental conditions. ${ }^{*} p<0.001$.

24-72 h p.i. (Fig. 4) and induced expression of iNOS in parasitized (Fig. 1G) and F4/80-positive macrophages (Fig. 1F), allowing us to confirm the source of iNOS derived NO in our system.

\section{Macrophage-mediated neuronal damage requires iNOS activity.}

In order to evaluate the role of $\mathrm{NO}$ in neuronal death in this model, we isolated macrophages from $\mathrm{iNOS}^{-/}$mice and repeated the experiments above. Addition of $\mathrm{iNOS}^{-/-}$macrophages alone did not interfere with neuronal survival. Indeed, infection of $\mathrm{iNOS}^{-/-}$macrophages with $T$. cruzi and activation with IFN- $\gamma$ in co-cultures failed to reproduce the neuronal death seen with WT cells (Fig. 5A), with no significant morphological alterations observed when compared to the control. As expected, NO production was not significant at all time points studied (Fig. 4). Further, the neurite density was similar to control (data not shown).

These results strongly indicated a critical role for IFN- $\gamma$ induced nitric oxide in neuronal damage, and this was assessed further through the pharmacological inhibition of iNOS in WT macrophages using aminoguanidine $(100 \mu \mathrm{M})$, which reduced NO amounts to an undetectable level (Fig. 5B) and failed to reproduce the neuronal death (Fig. 5A). We conclude that NO production from activated macrophages is a primary mechanism of neuronal damage in this model.

\section{Discussion}

We have developed a tissue culture model for the analysis of the cellular mechanisms responsible for the neuronal damage that occurs upon infection with the protozoan parasite, T. cruzi. 
Sympathetic neurons maintained in vitro with NGF survive and develop well. In our system, the incubation of SCG neuron and macrophage co-cultures with $T$. cruzi infective forms (trypomastigotes) resulted, as expected, in efficient macrophage invasion, but neurons were systematically non-infected, regardless of the
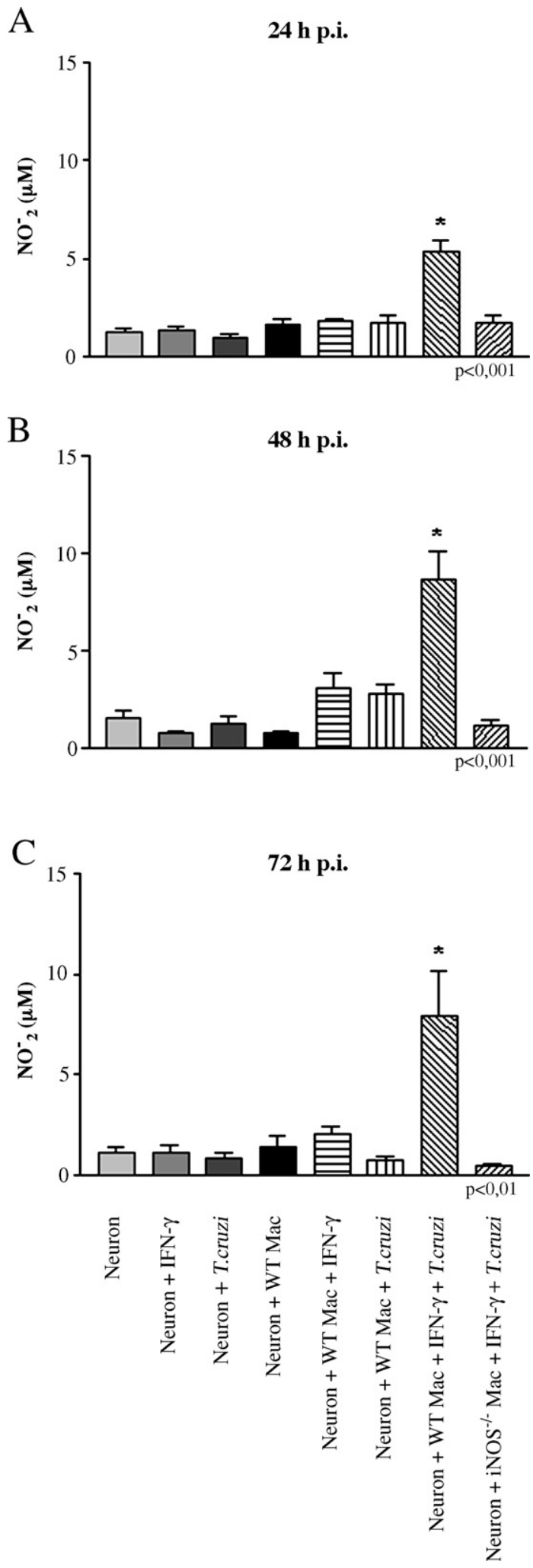

presence or absence of concomitant IFN- $\gamma$ treatment. The percentage of surviving neurons was not altered in pure neuronal cultures exposed to T. cruzi for $48 \mathrm{~h}$ (Fig. 2B) when compared to controls. In addition, this experimental condition did not result in NO production by 24, 48 and $72 \mathrm{~h}$ p.i. (Fig. 4). The addition of IFN- $\gamma$ and $T$. cruzi to pure neuronal cultures did not change the parameters described above (data not shown), nor the morphological aspect of these cultures when compared to the control cultures. Our findings suggest that neurons, in contrast to most mammalian cells, are resistant to parasite invasion. Our in vitro findings are in agreement with intensive and thorough studies that have shown a very small number of parasitized cells among the hundreds of thousands of ganglion cells in mice, rats and dogs experimentally infected with $T$. cruzi (Köberle, 1968). The mechanisms of neuronal resistance to $T$. cruzi invasion are currently under investigation.

We found that addition of normal/resting macrophages did not substantially alter neuronal survival, but that addition of a similar number of macrophages and the following concomitant infection with $T$. cruzi and activation by IFN- $\gamma$ led to significant and reproducible neurite loss and neuronal cell death. This clearly identifies the interaction between the macrophage and T. cruzi as a specific primary cause of neuronal damage, potentially responsible for the progressive neuronal damage and motility impairment seen in Chagas' disease.

Although iNOS is not considered essential for the control of T. cruzi infection (Cummings and Tarleton, 2004), it has been shown that resistance to $T$. cruzi is associated with the activation of macrophages by lymphocyte-derived IFN- $\gamma$ to produce nitric oxide. NO is paradoxically implicated in controlling intracellular T. cruzi multiplication (Gazzinelli et al., 1992; Vespa et al., 1994) but also plays a role in the tissue damage seen in Chagas' disease (Garcia et al., 1999; Vespa et al., 1994; Ny et al., 1999; Pinto et al., 2002; Chandra et al., 2002).

We focused on iNOS-derived NO as the important mediator of neuronal lesions seen in our model. This unique co-culture model reproduces the in vivo proximity and interaction between neurons and macrophages. SCG neurons in culture display eletrophysiological and molecular characteristics that resemble those in vivo (Blennerhassett and Bienenstock, 1998; Janiszewski et al., 1990). Our observation of the co-cultures showed that the macrophages were dispersed uniformly, representing ratio of approximately 10 macrophages per neuron. We believe that the levels of NO and subsequent neuronal damage obtained here are expected to mimic the conditions of natural T. cruzi infection. The neuronal loss of $60 \%$ at $72 \mathrm{~h}$ p.i. obtained in our study is in accordance with the myenteric neuronal loss described in experimental models (Arantes et al., 2004) and human studies (Köberle, 1968; Adad et al., 2001). Neuronal death is mediated by one or several interlinked deathinitiating pathways resulting in activation of two major final

Fig. 4. Increased $\mathrm{NO}^{-2}$ production in T. cruzi-infected and IFN- $\gamma$-activated co-cultures of neurons and WT macrophages (Neuron + WT Mac $+\mathrm{IFN}-\gamma+T$. cruzi) compared to T. cruzi-infected and IFN- $\gamma$-activated co-cultures of neurons and $\mathrm{iNOS}^{-/-}$macrophages (Neuron $+\mathrm{iNOS}^{-/-} \mathrm{Mac}+\mathrm{IFN}-\gamma+T$. cruzi) at 24 (A), 48 (B) and 72 (C) h p.i. We did not detect significant $\mathrm{NO}^{-2}$ production in controls (Neuron, Neuron + IFN- $\gamma$ ) nor experimental WT conditions (Neuron + T. cruzi, Neuron + WT Mac, Neuron+WT Mac+IFN$\gamma$, Neuron+WT Mac $+T$. cruzi). $N=3$ independent experiments in triplicates per experimental conditions. ${ }^{*} p<0.001$ (A, B) and ${ }^{*} p<0.01$ (C). 

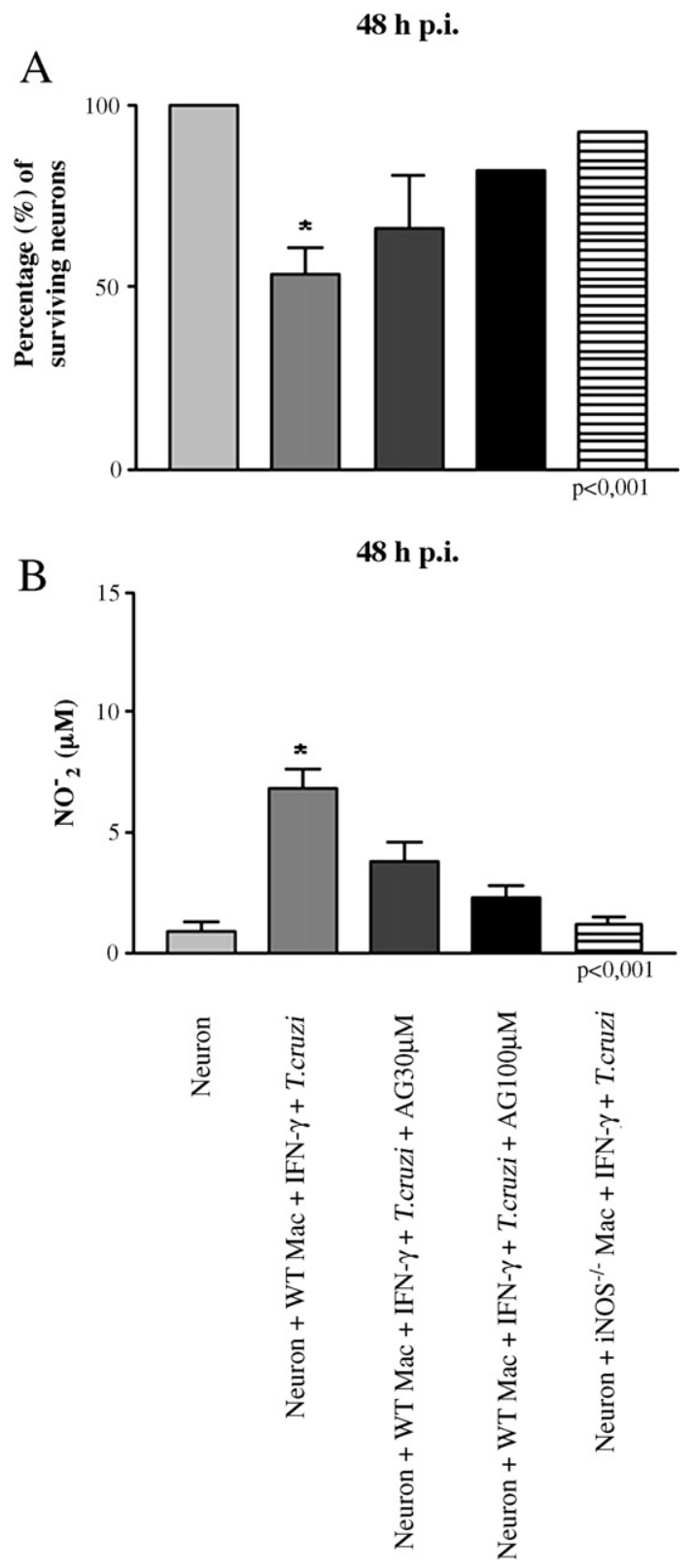

Fig. 5. Neuronal survival (A) and $\mathrm{NO}^{-2}$ production (B) in T. cruzi-infected and IFN- $\gamma$-activated co-cultures of neurons and WT or iNOS ${ }^{-/}$ macrophages. Recovered neuronal survival rate (A) and impaired $\mathrm{NO}^{-2}$ production (B) in aminoguanidine-treated T. cruzi-infected and IFN- $\gamma$ activated WT macrophages (Neuron $+\mathrm{WT}$ Mac $+\mathrm{IFN}-\gamma+T$. cruzi $+\mathrm{AG}$ $100 \mu \mathrm{M})$ and T. cruzi-infected and IFN- $\gamma$-activated $\mathrm{iNOS}^{-/-}$macrophages (Neuron $+\mathrm{iNOS}^{-/-} \mathrm{Mac}+\mathrm{IFN}-\gamma+T$. cruzi) compared to T. cruzi-infected and IFN- $\gamma$-activated WT macrophages (Neuron $+\mathrm{WT}$ Mac $+\mathrm{IFN}-\gamma+T$. cruzi) cocultures at 48 h p.i. $N=4$ independent experiments in triplicates. ${ }^{*} p<0.001$.

common pathways that lead to cell death (Leist and Jäättelä, 2001). NO-mediated neuronal damage has been described in many pathological conditions (Wang et al., 2002; Carreno-Muller et al., 2003; Eskes et al., 2003; Eyupoglu et al., 2003; Katsuse et al., 2003; Liu et al., 2003 Rozenfeld et al., 2003) and involves apoptosis (McCarthy et al., 1997; Stefanis et al., 1998; Yu et al., 2003). Necrosis is a catastrophic loss of ionic integrity caused by membrane disruption or loss of energy supply and was morphologically detected in association with apoptosis contributing to decreased neuronal survival in our model.

The in vivo source of IFN- $\gamma$ in natural infection is lymphocytes (Gazzinelli et al., 1992; Vespa et al., 1994). In our system, we provided IFN- $\gamma$ directly to the $T$. cruzi-infected macrophages reproducing the mechanism of macrophage activation described elsewhere (Drapier et al., 1988). The addition of IFN- $\gamma$ to T. cruziinfected co-cultures of neurons and macrophages increases NO production by macrophages between 24 and $72 \mathrm{~h}$ p.i. Detection of enhanced levels of nitrite, a stable degradation product of NO, and macrophage iNOS expression are correlated to significant neuronal damage assessed as decreased neuronal survival and decreased neurite density. The inability for NO production by $\mathrm{iNOS}^{-/}$macrophages was able to prevent development of neuronal damage observed in our system 24 to $72 \mathrm{~h}$ p.i., confirming the role of NO in the neuronal death in this model of $T$. cruzi-infected and IFN- $\gamma$-activated co-cultures. Neuronal death was additionally prevented by aminoguanidine, a specific iNOS inhibitor (Misko et al., 1993).

Although we have earlier experimental indirect evidence associating tissue damage with inflammation in mice in an experimental model of Chagas' disease (Arantes et al., 2004), this is the first demonstration of a direct effect of macrophage-derived NO in T. cruzi-induced neuronal damage. Besides macrophages, in $T$. cruzi and other infections, NO is produced by a wide variety of cell types, including NK cells, dendritic cells, neutrophils, endothelial cells, myocytes and fibroblasts (Hibbs et al., 1987; Ding et al., 1988; Kilbourn and Belloni, 1999; Werner-Felmayer et al., 1990; Nussler and Billiar, 1993; Vespa et al., 1994). During in vivo $T$. cruzi infection, different leukocyte sub-types are present in the inflammatory infiltrate, affecting tissue integrity. This makes the discrimination of the cellular sources of cytotoxic mediators to neural tissue difficult. Furthermore, in the in vivo model (Arantes et al., 2004), we could not also rule out the participation of glia cells as source of NO. In the current in vitro model, we eliminated viable glia cells in order to obtain pure neuronal cultures. If present, remaining glia represented less than $5 \%$ of cells and contribute to non-significant levels of $\mathrm{NO}$ that were detected in pure neuronal controls (Fig. 4), indicating that in this system glia cells should not be considered a relevant source of NO. The co-culturing of SCG neurons and WT macrophages for 24,48 and $72 \mathrm{~h}$ showed, respectively, $95 \%, 81 \%$ and $81 \%$ of neuronal survival related to controls confirming our previous results (Arantes et al., 2000). These degrees of loss of neuron survival and the NO levels measured in this condition are not significant when compared to control. On the other hand, NO is being generated in small amounts in the co-culture conditions (Neuron + Mac, Neuron $+\mathrm{Mac}+\mathrm{IFN}-\gamma$ or Neuron $+\mathrm{Mac}+T$. cruzi) as attested by Fig. 4. In agreement with our arguments, this may contribute to some degree for decreased, though not statistically significant, neuronal viability showed in these conditions, in Fig. 2. However, this contribution is not as significant as T. cruziinfected and IFN- $\gamma$-activated co-cultures.

We were able to correlate the enhanced levels of NO production not only with decreased neuronal survival but with neurite damage as well. Although ganglion cells are well visualized in murine and in human intestinal walls, the nerves are not routinely observed without specific staining. This can explain the focus of relevant investigations in counting neuronal body numbers (Adad et al., 2001; da Silveira et al., 2005, Köberle, 1970) without addressing axonal damage. In the context of Chagas' disease, however, the 
participation of inflammatory-induced axonal damage and synaptic alterations in the pathogenesis of motility disturbances and mega visceral syndromes are highly relevant and deserve further investigation. Acute and chronic damaging mechanisms could affect diverse regions of the ganglion cells, including the axon and its functional synapses, and could reflect the different degrees of dysfunctions clinically reported (Madigan et al., 1996; Meneghelli, 2004). One of the merits of this in vitro approach is to highlight the importance of studying the nerve-effector cells interaction in Chagas' disease, which we intend to pursue.

In conclusion, our findings add to the understanding of the mechanisms involved in the development of cardiac/digestive pathologies and contribute new insights into prevention and treatment of symptomatic forms of Chagas' disease.

\section{Acknowledgments}

This work was supported by grants from "UNDP/World Bank/ WHO Special Programme for Research and Training in Tropical diseases", Fundação de Amparo à Pesquisa do Estado de Minas Gerais-FAPEMIG (CDS195/02 and CBB1048/05). R.M.E.Arantes, F.Q. Cunha and L.M.C Galvão are supported by research fellowships from Conselho Nacional de Desenvolvimento Cientifico e Tecnológico (CNPq) and C.M.Almeida-Leite receives scholarship from Coordenação de Aperfeiçoamento de Pessoal do Ensino Superior (CAPES). The authors are indebted to Afonso C. Viana for maintaining the T. cruzi cultures and to L. Q. Vieira for IFN- $\gamma$ donation.

\section{References}

Adad, S.J., Cancado, C.G., Etchebehere, R.M., Teixeira, V.P., Gomes, U.A., Chapadeiro, E., Lopes, E.R., 2001. Neuron count reevaluation in the myenteric plexus of chagasic mega colon after morphometric neuron analysis. Virchows Arch. 438 (3), 254-258.

Alvarenga, R.J., 1960. Lesões do tecido adiposo na fase aguda da doença de Chagas experimental em camundongos (Inaugural Dissertation). Minas Gerais, University of Minas Gerais, School of Medicine, Belo Horizonte.

Arantes, R.M.E., Lourenssen, S., Machado, C.R.S., Blennerhassett, M.G., 2000. Early damage of sympathetic neurons after co-culture with macrophages: a model of neuronal injury in vitro. NeuroReport 11 (1), $177-181$.

Arantes, R.M.E., Marche, H.H., Bahia, M.T., Cunha, F.Q., Rossi, M.A., Silva, J.S., 2004. Interferon-gamma-induced nitric oxide causes intrinsic intestinal denervation in Trypanosoma cruzi-infected mice. Am. J. Pathol. 164 (4), 1361-1368.

Blennerhassett, M.G., Bienenstock, J., 1998. Sympathetic nerve contact causes maturation of mast cells in vitro. J. Neurobiol. 35, 173-182.

Braga, E.M., Galvão, L.M.C., Chiari, E., Martins, M.S., 1993. Difference in susceptibility to lysis between clones of the Y strain of Trypanosoma cruzi. Mem. Inst. Oswaldo Cruz 88 (4), 529-534.

Carreno-Muller, E., Herrera, A.J., De Pablos, R.M., Tomas-Camardiel, M., Venero, J.L., Cano, J., Machado, A., 2003. Thrombin induces in vivo degeneration of nigral dopaminergic neurones along with the activation of microglia. J. Neurochem. 84 (5), 1201-1214.

Chandra, M., Tanowitz, H.B., Petkova, S.B., Huang, H., Weiss, L.M., Wittner, M., Factor, S.M., Shtutin, V., Jelicks, L.A., Chan, J., Shirani, J., 2002. Significance of inducible nitric oxide synthase in acute myocarditis caused by Trypanosoma cruzi (Tulahuen strain). Int. J. Parasitol. 32, 897-905.

Cummings, K.L., Tarleton, R.L., 2004. Inducible nitric oxide synthase is not essential for control of Trypanosoma cruzi infection in mice. Infect. Immun. 72 (7), 4081-4089.

da Silveira, A.B., Arantes, R.M., Vago, A.R., Lemos, E.M., Adad, S.J., Correa-Oliveira, R., D'Avila Reis, D., 2005. Comparative study of the presence of Trypanosoma cruzi kDNA, inflammation and denervation in chagasic patients with and without megaesophagus. Parasitology 131 (Pt 5), 627-634.

Ding, A.H., Nathan, C.F., Stuehr, D.J., 1988. Release of reactive nitrogen intermediates and reactive oxygen intermediates from mouse peritoneal macrophages. Comparison of activating cytokines and evidence for independent production. J. Immunol. 141, 2407-2412.

Drapier, J.C., Wietzerbin, J., Hibb, J.B., 1988. Interferon-gamma and tumor necrosis factor induce the L-arginine dependent cytotoxic effector mechanism in murine macrophages. Eur. J. Immunol. 18, 1587-1592.

du Manoir, J.M., Albright, B.N., Stevenson, G., Thompson, S.H., Mitchell, G.B., Clark, M.E., Caswell, J.L., 2002. Variability of neutrophil and pulmonary alveolar macrophage function in swine. Vet. Immunol. Immunopathol. 89 (3-4), 175-186.

Eskes, C., Juillerat-Jeanneret, L., Leuba, G., Honegger, P., Monnet-Tschudi, F., 2003. Involvement of microglia-neuron interactions in the tumor necrosis factor-alpha release, microglial activation, and neurodegeneration induced by trimethyltin. J. Neurosci. Res. 71 (4), 583-590.

Eyupoglu, I.Y., Bechmann, I., Nitsch, R., 2003. Modification of microglia function protects from lesion-induced neuronal alterations and promotes sprouting in the hippocampus. FASEB J. 17 (9), 1110-1111.

Garcia, S.B., Paula, J.S., Giovanetti, G.S., Zenha, F., Ramalho, E.M., Zucoloto, S., Silva, J.S., Cunha, F.Q., 1999. Nitric oxide is involved in the lesions of the peripheral autonomic neurons observed in the acute phase of experimental Trypanosoma cruzi infection. Exp. Parasitol. 93, 191-197.

Gazzinelli, R.T., Oswald, I.P., Hieny, S., James, S.L., Sher, A., 1992. The microbicidal activity of interferon-gamma-treated macrophages against Trypanosoma cruzi involves an L-arginine-dependent, nitrogen oxidemediated mechanism inhibitable by interleukin-10 and transforming growth factor-beta. Eur. J. Immunol. 22 (10), 2501-2506.

Green, L.C., Luzuriaga, K.R., Wagner, D.A., Rand, W., Instan, N., Young, V.R., Tannenbaum, S.R., 1981. Nitrate biosynthesis in man. Proc. Natl. Acad. Sci. U. S. A. 78, 7764-7768.

Hibbs Jr., J.B., Taintor, R.R., Vavrin, Z., 1987. Macrophage cytotoxicity: role for $\mathrm{L}$-arginine deaminase and immino nitrogen oxidation to nitrite. Science 235, 473-476.

Janiszewski, J., Bienenstock, J., Blennerhassett, M.G., 1990. Activation of rat peritoneal mast cells in coculture with sympathetic neurons alters neuronal physiology. Brain Behav. Immun. 4 (2), 139-150.

Katsuse, O., Iseki, E., Kosaka, K., 2003. Immunohistochemical study of the expression of cytokines and nitric oxide synthases in brains of patients with dementia with Lewy bodies. Neuropathology 23 (1), $9-15$.

Kilbourn, R.G., Belloni, P., 1999. Endothelial cell production of nitrogen oxides in response to interferon gamma in combination with tumor necrosis factor, interleukin-1, or endotoxin. J. Natl. Cancer Inst. 82 (9), $772-776$.

Köberle, F., 1956. Über das neurotoxin des Trypanosoma cruzi. Zbl Allg Path. Anat. 95, 468-475.

Köberle, F., 1968. Chagas' disease and Chagas' syndromes: the pathology of American trypanosomiasis. Adv. Parasitol. 6, 63-116.

Köberle, F., 1970. The causation and importance of nervous lesions in American trypanosomiasis. Bull. WHO 42, 739-743.

Leist, M., Jäättelä, M., 2001. Four deaths and a funeral: from caspases to alternative mechanisms. Nat. Rev., Mol. Cell Biol. 2, 589-598.

Liu, Y., Qin, L., Li, G., Zhang, W., An, L., Liu, B., Hong, J.S., 2003. Dextromethorphan protects dopaminergic neurons against inflammation-mediated degeneration through inhibition of microglial activation. J. Pharmacol. Exp. Ther. 305 (1), 212-218.

MacClure, E., Poche, R., 1960. Die experimentelle Chagas-Myokarditis bei der weien Maus im elektonenmikroskopischen. Bild. Virchows Arch. $333,405-420$. 
Madigan, M.C., Sadun, A.A., Rao, N.S., Dugel, P.U., Tenhula, W.N., Gill, P.S., 1996. Tumor necrosis factor-alpha (TNF-alpha)-induced optic neuropathy in rabbits. Neurol. Res. 18 (2), 176-184.

McCarthy, M.J., Rubin, L.L., Philpott, K.L., 1997. Involvement of caspases in sympathetic neuron apoptosis. J. Cell Sci. 110 (Pt 18), 2165-2173.

Meneghelli, U.G., 2004. Chagasic enteropathy. Rev. Soc. Bras. Med. Trop. 37 (3), 252-260

Misko, T.P., Moore, W.M., Kasten, T.P., Nickols, G.A., Corbett, J.A., Tilton, R.G., McDaniel, M.L., Williamson, J.R., Currie, M.G., 1993. Selective inhibition of the inducible nitric oxide synthase by aminoguanidine. Eur. J. Pharmacol. 233 (10), 119-125.

Moncayo, A., 2003. Chagas' disease: current epidemiological trends after the interruption of vectorial and transfusional transmission in the Southern Cone countries. Mem. Inst. Oswaldo Cruz 98 (5), 577-591.

Nathan, C., Shiloh, M.U., 2000. Reactive oxygen and nitrogen intermediates in the relationship between mammalian hosts and microbial pathogens. Proc. Natl. Acad Sci. U. S. A. 97 (16), 8841-8848.

Nathan, C., Xie, Q.W., 1994. Nitric oxide synthases: roles, tolls, and controls. Cell 78 (6), 915-918.

Ny, L., Persson, K., Larsson, B., Chan, J., Weiss, L.M., Wittner, M., Huang, H., Tanowitz, H.B., 1999. Localization and activity of nitric oxide synthases in the gastrointestinal tract of Trypanosoma cruzi-infected mice. J. Neuroimmunol. 99, 27-35.

Nussler, A.K., Billiar, T.R., 1993. Inflammation, immunoregulation, and inducible nitric oxide synthase. J. Leukocyte Biol. 54, 171-178.

Okumura, M. 1966. Contribuição para o estudo das lesões dos neurônios do plexo mientérico do colon na Moléstia de Chagas experimental no camundongo branco (Mus musculus L.). PhD thesis. Faculty of Medicine, University of São Paulo, São Paulo.

Pinto, N.X., Torres-Hillera, M.A., Mendoza, E., Leon-Sarmiento, F.E., 2002. Immune response, nitric oxide, autonomic dysfunction and stroke: a puzzling linkage on Trypanosoma cruzi infection. Med. Hypotheses $58,374-377$.
Rossi, M.A., Bestetti, R.B., 1995. The challenge of Chagas' cardiomyopathy. The pathologic roles of autonomic abnormalities, autoimmune mechanisms and microvascular changes, and therapeutic implications. Cardiology 86, 01-07.

Rozenfeld, C., Martinez, R., Figueiredo, R.T., Bozza, M.T., Lima, F.R., Pires, A.L., Silva, P.M., Bonomo, A., Lannes-Vieira, J., De Souza, W., Moura-Neto, V., 2003. Soluble factors released by Toxoplasma gondiiinfected astrocytes down-modulate nitric oxide production by gamma interferon-activated microglia and prevent neuronal degeneration. Infect. Immun. 71 (4), 2047-2057.

Stefanis, L., Troy, C.M., Qi, H., Shelanski, M.L., Greene, L.A., 1998. Caspase-2 (Nedd-2) processing and death of trophic factor-deprived PC12 cells and sympathetic neurons occur independently of caspase-3 (CPP32)-like activity. J. Neurosci. 18 (22), 9204-9215.

Stuehr, D.J., 1999. Mammalian nitric oxide synthases. Biochim. Biophys. Acta $1411(2-3), 217-230$

Tafuri, W.L., 1970. Pathogenesis of lesions of the autonomic nervous system of the mouse in experimental acute Chagas' disease. Light and electron microscope studies. Am. J. Trop. Med. Hyg. 19 (3), 405-417.

Vespa, G.N., Cunha, F.Q., Silva, J.S., 1994. Nitric oxide is involved in control of Trypanosoma cruzi-induced parasitemia and directly kills the parasite in vitro. Infect. Immun. 62, 5177-5182.

Wang, M.J., Lin, W.W., Chen, H.L., Chang, Y.H., Ou, H.C., Kuo, J.S., Hong, J.S., Jeng, K.C., 2002. Silymarin protects dopaminergic neurons against lipopolysaccharide-induced neurotoxicity by inhibiting microglia activation. Eur. J. Neurosci. 16 (11), 2103-2112.

Werner-Felmayer, G., Werner, E.R., Fuchs, D., Hausen, A., Reibnegger, G., Wachter, H., 1990. Tetrahydrobiopterin-dependent formation of nitrite and nitrate in murine fibroblasts. J. Exp. Med. 172 (6), 1599-1607.

Yu, L.Y., Jokitalo, E., Sun, Y.F., Mehlen, P., Lindholm, D., Saarma, M., Arumae, U., 2003. GDNF-deprived sympathetic neurons die via a novel nonmitochondrial pathway. J. Cell Biol. 163 (5), 987-997. 